\title{
Predictor-Corrector Entry Guidance for Reusable Launch Vehicles ${ }^{\dagger}$
}

\author{
Dr. Hussein Youssef $\$$ \\ Dr. Rajiv Chowdhry \\ Howard Lee \\ Lockheed Martin Aeronautics Company-Palmdale \\ Palmdale, CA 93599 \\ Curtis Zimmerman ${ }^{*}$ \\ NASA Marshall Space Flight Center \\ Huntsville, AL 35812
}

\begin{abstract}
An online entry guidance algorithm has been developed using a predictor-corrector approach. The algorithm is designed for the Reusable Launch Vehicle (RI,V) and is demonstrated by using the X33 model. The objective of the design is to handle widely dispersed entry conditions and deliver the vehicla at the Terminal Area Energy Management (TAEM) interface box within an acceptable tolerance and without violating any of the vehicle physical constraints. Combination of several control variables is used in testing the performance and computational requirement of the algorithm. The control variables are the bank angle, angle-of-attack and the time for roll reversal. The bank angle and angle-of-attack profiles are the nominal profiles plus the perturbations in each direction. The initial guess of the bank profile is a 45 degrees bank angle with reversal at 360 sezonds from liftoff. A six-element state vector is propagated to the TAEM interface box through the integration of the equations of motion (EOM). Altitude, heading and range errors are computed between the desired and the achieved state at the TAEM interface. These errors are used to correct the initial guess of the control variables. This process is repeated until the errors meet an acceptable level at the TAEM interface. Several numerical optimization methods are used to evaluate the convergent property of the predictor-predictor methodology. Successful results are demonstrated using the X-33 model.
\end{abstract}

\section{BACKGROUND}

An entry guidance algorithm based on the Space Shuttle drag-energy modulation approach has been developed for a RLV and demonstrated using the $X$ 33 model. This guidance algorithm works well in terms of handling dispersions and successfully flying to TAEM interface while meeting the thermal, structural, and control power constraints. However, the thermal constraints for a metallic TPS (being used on $\mathrm{X}-33$ RLV vehicle to reduce weight and improve operability) are different than those used for the Space Shuttle TPS. Also, the Shuttle-based guidance law uses gain-scheduled parameters, which requires off-line tuning for every mission. Since the gains are fixed for a flight, the guidance law only handles preplanned contingencies and lacks the adaptability to accommodate unforeseen circumstances during flight. The costs associated with this labor intensive approach can be substantial.

The limitations of the existing guidance algorithm caused NASA MSFC to launch the Advanced Guidance and Control (AG\&C) project [1] in April 1999 with completion planned in March 2001. Different NASA centers and universities are developing several competing guidance algorithms. The goal is to determine, via simulation fly-off, which method has the highest potential on reducing the operational cost and increasing the mission safety of the RLV. Lockheed Martin Aeronautics - Palmdale (the Skunk Works) worked closely with NASA MSFC in defining the requirements for these algorithms. The candidate algorithms for ascent and

\footnotetext{
${ }^{\dagger}$ This paper acknowledges NASA's support under Cooperative Agreement NCC8-115.

${ }^{5}$ Technical Fellow, Sr. Member AIAA

- Research and Development Engineer, Member AIAA

- Aerospace Engineer, Member AIAA
} 
entry being considered are the hybrid ascent/abort guidance using optimal control and collocation techniques, ascent/abort guidance scheme based on Neighboring Optimal Control (NOC) techniques, trajectory following ascent guidance scheme based on continuous-time predictive control concept, predictor corrector entry guidance techniques, entry guidance by trajectory regulations about a reference trajectory and reduced order predictive/tracking entry guidance. This paper concerns the predictor-corrector entry guidance only

\section{INTRODUCTION}

The goal of the second generation RLV program is to reduce the cost of placing the payload in orbit by an order of magnitude and the probability of loss of vehicle by two orders of magnitude relative to current Space Shuttle operations today. The objective of reducing the payload cost to $\$ 1000 / \mathrm{lb}$ and the loss of the vehicle to $I$ in 10,000 is requiring the $A G \& C$ to be adaptive, robust, and reconfigurable. The proposed method in this paper will help to achieve the cost and reliability objectives.

The predictor-corrector algorithm described in this paper is demonstrated by using the X-33 model. The $\mathrm{X}-33$ is a subscale technology demonstrator for the RLV. A typical mission profile is shown in Figure 1. The predictor-corrector algorithm is applied to the descent portion down to the TAEM interface. The baseline entry guidance on the $\mathrm{X}-33$ is a Shuttle based algorithm with modification to accommodate the peculiarity of the X-33 design especially in the metallic Thermal Protection System (TPS) area [2].

The major advantage of the predictor-corrector method is that the EOM are used explicitly in the formulation of the algorithm. Thus the vehicle can be modeled in as much detail as needed. Having the state vector available every integration step allows the calculation of the TPS thermal stress indicator and its use as an active constraint. Other areas of concern are the real-time implementation and the rate of convergence to feasible solution.

Predictor-corrector algorithms have been evaluated for the past several years. Reference [3] describes numerical roll reversal predictor-corrector algorithm in aerocapture and precision landing guidance for the Mars Surveyor missions. Predictor-corrector algorithms have also been demonstrated for entry and landing missions. Reference [4] describes predictorcorrector scheme for a Space Station resupply/rescue vehicle. The remaining sections of this paper describe the general approach, algorithm developinent, optimization methods, and set of constraints, simulation results, conclusions, acknowledgements, and references.

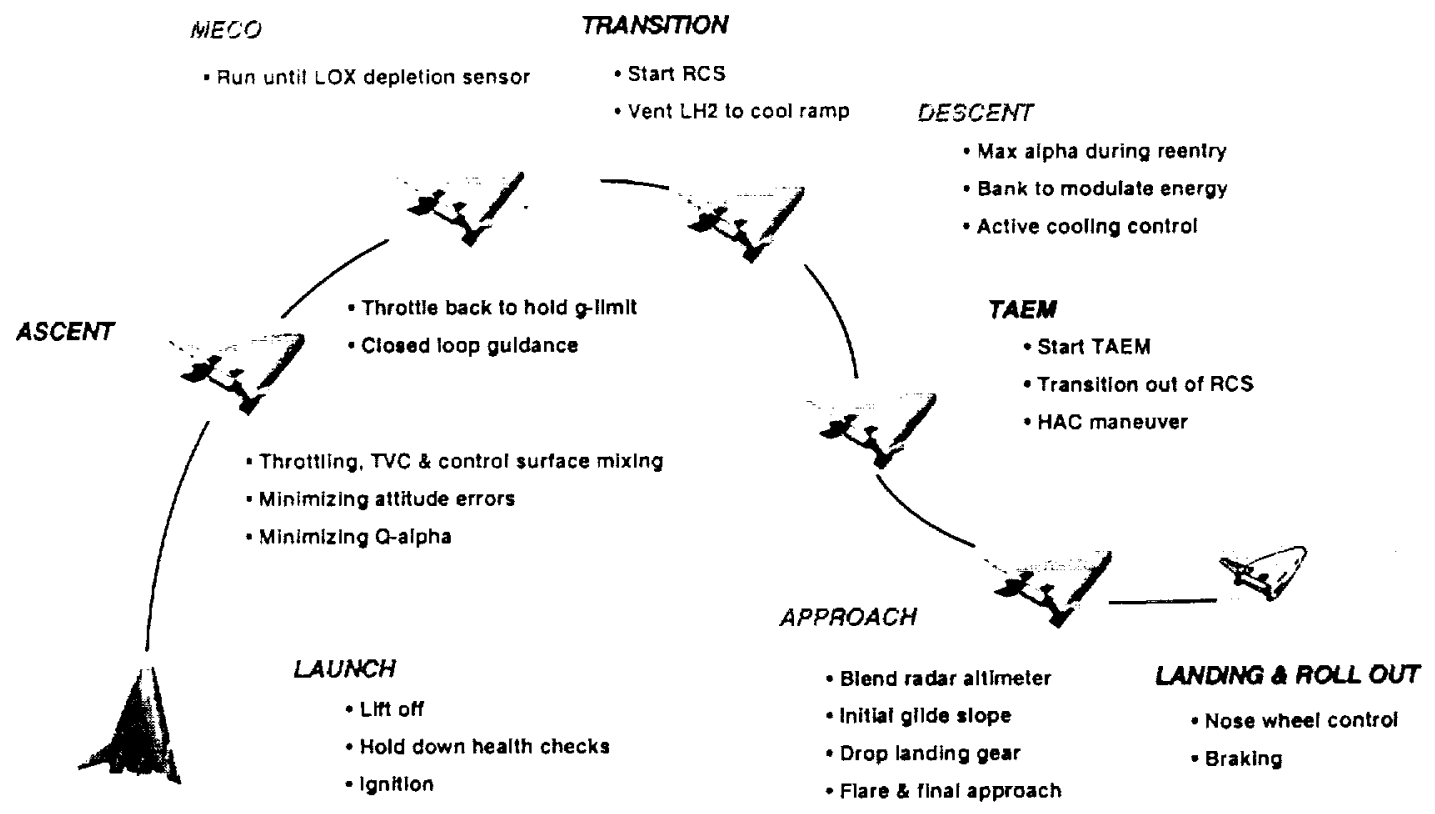

Figure 1 - X-33 Mission Profile 2

American Institute of Aeronautics and Astronautics 


\section{APPROACH}

The attractive reason for designing entry guidance algorithm on line is the ability to include the thermalstress indicator (TSI) as an active constraint. The TSI computation is a function of the temperature variation within the panels $(\Delta T)$ and the pressure variation $(\Delta P)$ across the TPS panel. This requires solving a simplified one-dimensional heat transfer equation through the panel. In addition, the TAEM interface box needs to be satisfied (i.e. altitude, heading, and down range.) Multiple steps approach is considered to start with simpler formulation to more elaborate scheme as we progress in achieving our goals.

The first approach is concentrated on achieving the TAEM interface using the bank angle as the only control variable. A six-element state vector is propagated using transnational EOM to the TAEM interface defined by a desired box. The difference between the propagated state and the desired state at the TAEM interface is defined as the error state. Several iterations in modifying the bank angle profile are used to generate the functional relationship between the error and bank angle command. Several optimization schemes are tried to find the control variable solution using MATLAB simulation.

The second step is introduced to allow for incorporating the TSI constraint. Additional control variables are needed to increase the degrees of freedom such that the TAEM interface and TSI constraints are met simultaneously. The nominal bank profile is described as a single doublet as shown in figure 2. The bank angle control variables are $\Delta \sigma_{1}$, \lrcorner$\sigma_{2}$ and $\Delta t$ changes for reversal time $t_{r}$. The nominal angle-of-attach profile is described as a three level step function as shown in figure 3 . The angle-ofattack control variables are $\Delta \alpha_{1}, \Delta \alpha_{2}, \Delta t_{1}$, and $\Delta t_{2}$.

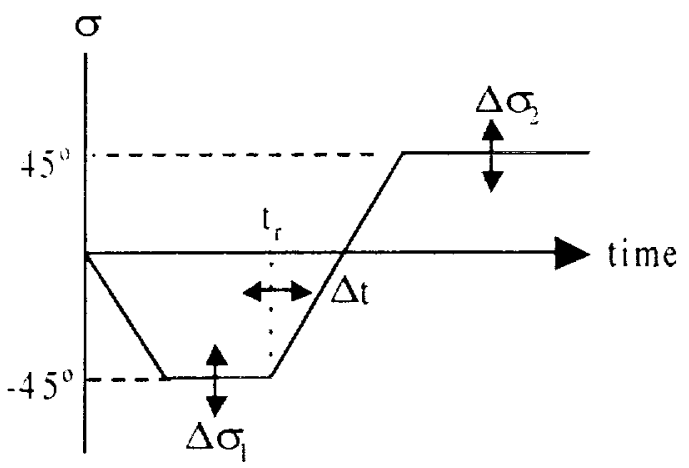

Figure 2 - Bank controlvariables

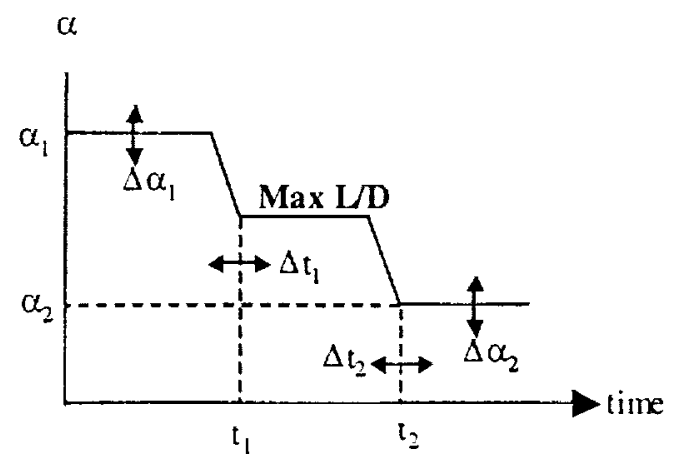

Figure $3-\alpha$ control variables

\section{ALGORITHMS DEVELOPMENT}

A key consideration to a successful predictorcorrector algorithm is establishing the sensitivity of the state error relative to the control variable and defining the performance index to solve for the control variable. Hundreds of trajectory solutions from entry to TAEM interface are generated to establish the functional relationships between the state error and control variables. Typical examples of heading error and down range error as function of $\Delta \sigma_{1}$ and $\Delta \sigma_{2}$ at a constant $t_{r}$ are shown in figures + and 5 , respectively.

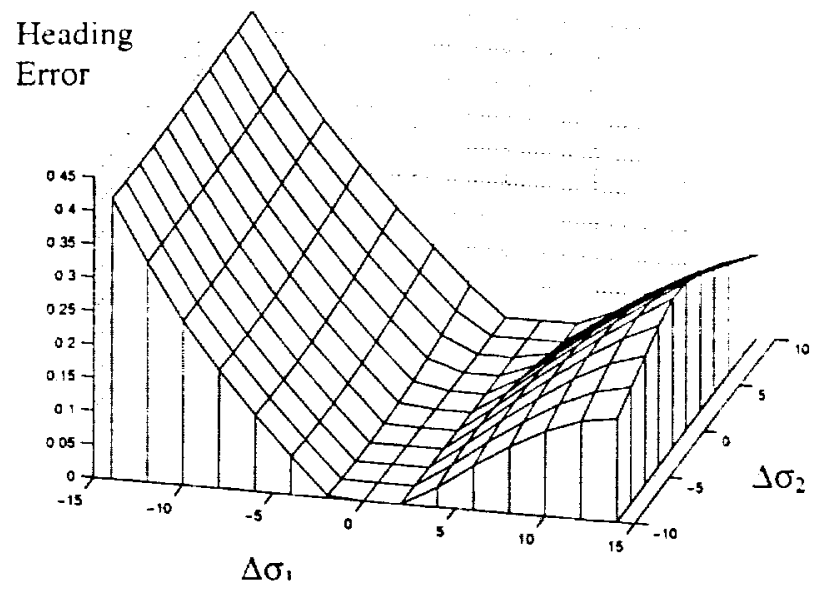

Figure 4 - Heading error as function of $\Delta \sigma_{1}$ and $\Delta \sigma_{2}$ 


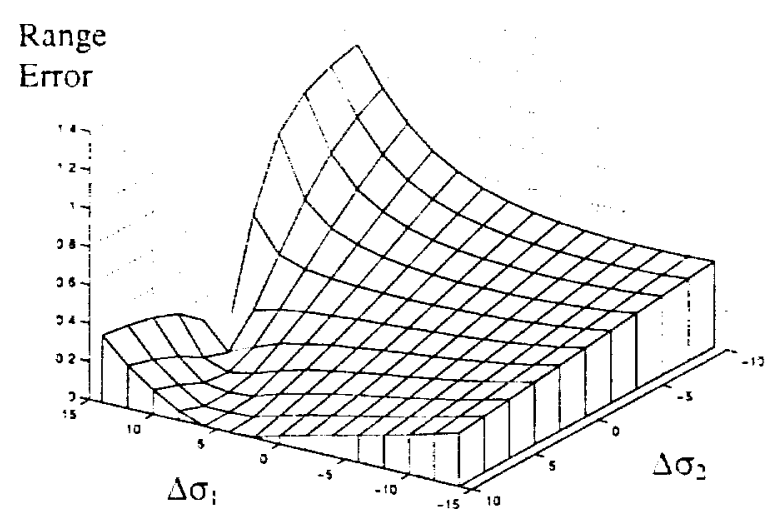

Figure 5 - Range error as function of $\Delta \sigma_{1}$ and $\Delta \sigma_{2}$

Several performance indices are considered in generating the control variables. They ranges from the straightforward approach of finding the zeros of the nonlinear function to minimizing a L2 norm of the combined errors. Consistent results are obtained using the later approach of minimizing the combined errors function $f$ with respect to the control variables, where:

$$
f=\sqrt{f_{1}^{2}+f_{2}^{2}+f_{3}^{2}}
$$

and

$$
\begin{aligned}
& f_{1}=\left(\frac{\Delta R_{e}}{S F_{1}}\right) \\
& f_{2}=\left(\frac{\Delta \chi_{e}}{S F_{2}}\right) \\
& f_{3}=\left(\frac{\Delta h_{e}}{S F_{3}}\right)
\end{aligned}
$$

$\Delta R_{\mathrm{e}}$ is the down range error,

$S F_{1}$ is the scale factor associated with $\Delta \mathrm{R}_{e}$ $\Delta \chi_{0}$ is the heading error
$S F_{2}$ is the scale factor associated with $\Delta \chi_{e}$ $\Delta h_{\mathrm{c}}$ is the altitude error

$S F_{3}$ is the scale factor associated with $\Delta h_{e}$

Although it is recognized that real time implementation is dependent on the convergence rate of the solution, more emphasis is placed on the consistency of finding the solution. There are several ways that can speed the convergence of the solution by using specially tailored optimization schemes coded in C rather than MATLAB.

\section{SET OF CONSTRAINTS}

The TPS constraints during entry are the primary focus of guidance algorithms. The shuttle-based entry guidance [5] using a drag-energy modulation can accommodate stagnation temperature and reference heat rate as shown in figure 6 . However, for metallic TPS panels the TSI constraint is the major concern. In order to implement that constraint, a one-dimensional heat transfer equation through the TPS panel is used. The input to that equation is the state vector evaluated at each time step of integrating the EOM. The output of that equation is the temperature differential across the TPS panel and the equilibrium wall temperature of the outer panel. Both these quantities are needed to calculate the TSI and the creep indicator (CI) of the panel. The solution of the heat transfer equation is repeated at different panels that represent critical locations over the vehicle. Figure 7 shows the different body points used in calculating thermal constraints and the layout of the TPS metallic panels. The structural constraints are represented by the bending moment, shear, and torsion at the root of the canted fins and vertical fins. The load indicator is a measure of the severity of the structural loads are the normal acceleration $\left(\mathrm{N}_{z}\right)$ and the product of dynamic pressure multiplied by angleof-attack $(Q \alpha)$.

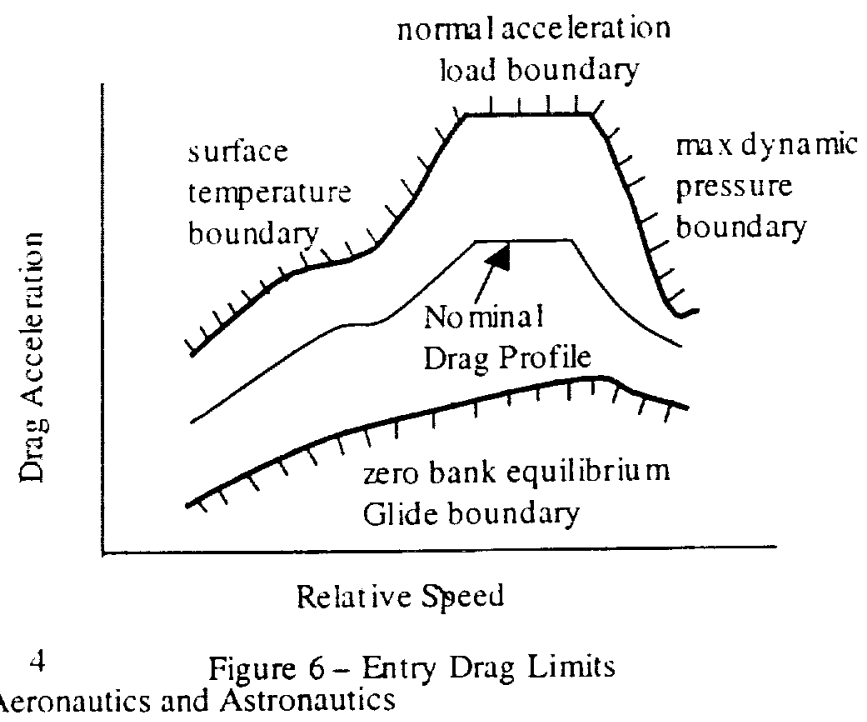

American Institute of Aeronautics and Astronautics 


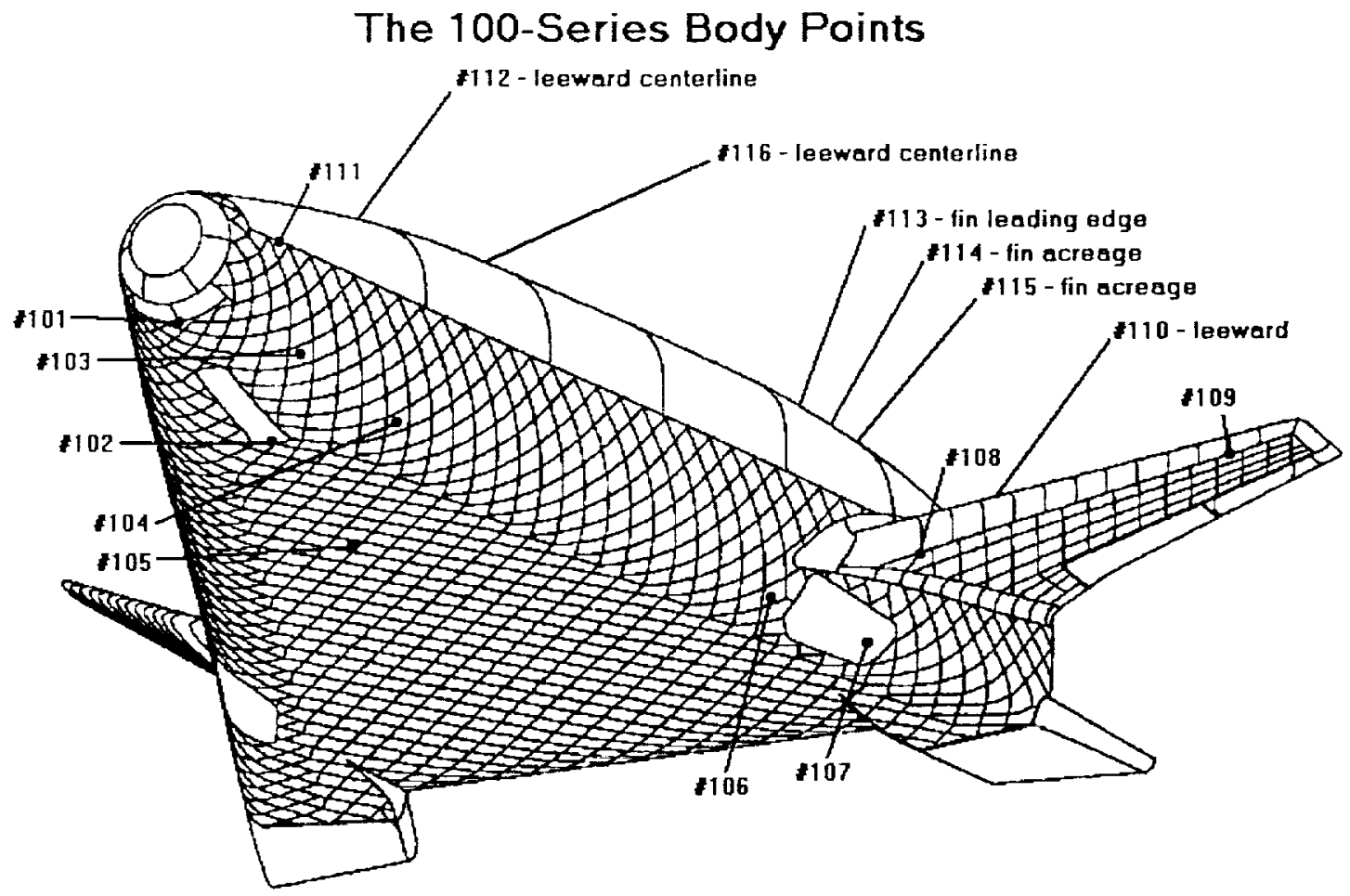

Figure 7 - Aerothermal constraint body points and TPS layout

The other set of significant constraints are defined at the TAEM interface box in terms of altitude, down range, and heading.

The number of control variables describing the angleof-attack profile and bank angle profile are more than or equal to the number of active constraints during the optimization.

\section{SIMLLATION RESULTS}

A 3-DOF MATLAB simulation for the $\mathrm{X}-33$ is used for the conceptual development of the predictorcorrector entry guidance. The simulation is validated against the MSFC test bed simulation called MAVERIC (Murshal Aerospace VEhicle Representation in C.) The propagation of the three transnational EOM is performed using the standard fourth order Runge-Kutta integration scheme. The optimization scheme to solve for the control variables subject to the set of constraints is the default option of MATLAB. Although it is recognized that the use of MATLAB simulation is not the most efficient way for real time computation, it was useful in determining the proof of concept. Several perturbed state cases for initial entry condition and different initial solution guesses are used to test the robustness of the predictor-corrector algorithm.

The first set of results is obtained using bank modulation only to meet the TAEM interface constraints of downrange and heading requirements as shown in table 1 . It was apparent that the altitude at TAEM interface was consistently on the low side. At that time the last step in the angle of attack profile $\left(\Delta \alpha_{2}\right)$ was introduced as an additional control variable. It was found that small change in $\Delta \alpha_{2}$ (in the order of 2 degrees) was enough to bias the TAEM altitude to be inside the desirable box. The last set of results is obtained using both bank and angle-ofattack modulation to meet the TAEM interface and TSI constraints as shown in table 2.

Figures 8 through 14 show the time responses for the low energy case $\left(\mathrm{V}_{0}-500 \mathrm{fps}\right)$ using 4 control variables as summarized in Table 2 . 
Table 1 - Predictor-Corrector guidance with 3 control parameters, minimize L2 norm

\begin{tabular}{|c|c|c|c|c|c|c|c|c|c|}
\hline & Nonimal & $\begin{array}{c}V_{0}+500 \\
\text { fps }\end{array}$ & $\begin{array}{c}V_{0}-500 \\
\text { fps }\end{array}$ & $\begin{array}{c}\mathrm{H}_{0}+10 \\
\mathrm{~K} \mathrm{ft} \\
\end{array}$ & $\begin{array}{c}\mathrm{H}_{0}-\mathrm{I0} \\
\mathrm{K} \mathrm{ft}\end{array}$ & $\begin{array}{c}\chi_{0}+2 \\
\operatorname{deg}\end{array}$ & $\begin{array}{l}\chi_{0}-2 \\
\text { deg }\end{array}$ & $\begin{array}{c}\gamma_{0}+0.5 \\
\text { deg }\end{array}$ & $\begin{array}{c}\gamma_{0}-0.5 \\
\text { deg }\end{array}$ \\
\hline Range Ertor, nm & 1.66 & -4.29 & $-2.64 !$ & 3.5 & -2.94 & 1.22 & 1.75 & -3.29 & 0.44 \\
\hline Azimuth error, deg & 2.32 & -2.73 & -3.89 & -1.61 & -2.37 & -3.75 & -3.69 & -0.46 & 3.87 \\
\hline TAEM altitude, $\mathrm{ft}$ & 91312.0 & 90796.0 & 91345.0 & 92791.0 & 92574.0 & 91089.0 & 91765.0 & 90850.0 & 91211.0 \\
\hline No. of iterations & 3 & 2 & 2 & 4 & 2 & 2 & 4 & 2 & 3 \\
\hline $\begin{array}{l}\text { No. of function } \\
\text { evaluations }\end{array}$ & 21 & 12 & 11 & 27 & 11 & 11 & 25 & II & 19 \\
\hline initial $\Delta \sigma_{1}$, deg & -5.00 & -12.00 & $12.00^{i}$ & -12.00 & -2.00 & -2.00 & 2.00 & -2.00 & -2.00 \\
\hline initial $\Delta \sigma_{2}$, deg & 5.00 & 5.00 & -5.00 & 5.00 & -5.00 & 5.00 & -5.00 & 5.00 & 2.00 \\
\hline initial $t_{1}, \mathrm{sec}$ & 360.00 & 340.00 & 340.00 & 340.00 & 350.00 & 350.00 & 350.00 & $3+0.00$ & 360.00 \\
\hline final $\Delta \sigma_{1}$, deg & -2.98 & -12.00 & 11.73 & -9.91 & -2.03 & -2.10 & -4.25 & -2.11 & -1.39 \\
\hline final $\Delta \sigma_{2}$, deg & 3.86 & 5.01 & -5.12 & 0.85 & -4.87 & 4.96 & 0.57 & +.96 & 0.70 \\
\hline tinal $t_{r}$, sec & 346.00 & 338.50 & 360.70 & 340.60 & 339.10 & 357.90 & 337.60 & 351.90 & 340.90 \\
\hline
\end{tabular}

Table 2 - Predictor-Corrector guidance with 4 control parameters, minize L2 norm

\begin{tabular}{|c|c|c|c|c|c|c|c|c|c|}
\hline & Nonimal & $\begin{array}{c}\mathrm{V}_{0}+500 \\
\mathrm{fps}\end{array}$ & $\begin{array}{c}V_{0^{-}} 500 \\
\text { fps }\end{array}$ & $\begin{array}{c}\mathrm{H}_{0}+10 \\
\mathrm{~K} \mathrm{n}\end{array}$ & $\begin{array}{c}\mathrm{H}_{0}-10 \\
\mathrm{~K} \mathrm{ft}\end{array}$ & $\begin{array}{l}\chi_{0}+2 \\
\operatorname{deg}\end{array}$ & $\begin{array}{l}\chi_{0^{-}}-2 \\
\operatorname{deg}\end{array}$ & $\begin{array}{c}\gamma_{0}+0.5 \\
\mathrm{deg}\end{array}$ & $\begin{array}{l}\gamma_{0}-0.5 \\
\operatorname{deg}\end{array}$ \\
\hline Range Error, nm & 2.71 & -3.72 & -2.91 & 1.85 & -4.09 & 0.87 & 0.14 & -1.75 & 4.02 \\
\hline Azimuth error, deg & 4.34 & -2.55 & -2.04 & -1.94 & 2.53 & -1.92 & 4.39 & -4.58 & -1.69 \\
\hline TAEM altitude, $\mathrm{ft}$ & 93160.0 & 92557.0 & 93188.0 & 92603.0 & 92364.0 & $92+64.0$ & 92509.0 & 92363.0 & $928+4.0$ \\
\hline Max TSI 105 & 0.744 & 0.807 & 0.679 & 0.852 & 0.887 & $0.7+1$ & 0.734 & 0.723 & 0.766 \\
\hline No. of iterations & 3 & 2 & 2 & 6 & 2 & 2 & 5 & 3 & 4 \\
\hline $\begin{array}{l}\text { No. of function } \\
\text { evaluations }\end{array}$ & 23 & 13 & 13 & 42 & 13 & 13 & 44 & 22 & 23 \\
\hline initial $\Delta \sigma_{1}$, deg & -5.00 & -12.00 & 12.00 & -12.00 & -2.00 & -2.00 & 2.00 & -2.00 & -2.00 \\
\hline initial $\Delta \sigma_{2}$, deg & 5.00 & 5.00 & -5.00 & 5.00 & -5.00 & 5.00 & -5.00 & 5.00 & 2.00 \\
\hline initial $\Delta \alpha$, deg & 1.50 & 1.50 & 1.50 & 0.50 & 0.50 & 1.50 & 1.50 & 1.50 & 1.50 \\
\hline initial $t_{T}$, sec & 360.00 & 350.00 & 340.00 & 340.00 & 350.00 & 350.00 & 350.00 & 340.00 & 360.00 \\
\hline final $\Delta \sigma_{1}$, deg & -3.15 & -11.88 & 11.73 & -12.36 & -2.00 & -2.12 & 0.42 & -2.57 & -1.56 \\
\hline final $\Delta \sigma_{2}$, deg & 4.22 & 5.07 & -5.26 & -2.73 & -4.85 & 4.95 & 5.28 & 5.10 & 2.37 \\
\hline final $\Delta \alpha, \operatorname{deg}$ & 1.47 & 1.50 & 1.50 & 0.57 & 0.50 & 1.50 & 1.74 & $1,+2$ & 1.50 \\
\hline final tr, sec & $3+6.70$ & 339.80 & 360.90 & 336.70 & 337.90 & 358.00 & 344.00 & 354.30 & $3+6.50$ \\
\hline
\end{tabular}

6

American Institute of Aeronautics and Astronautics 


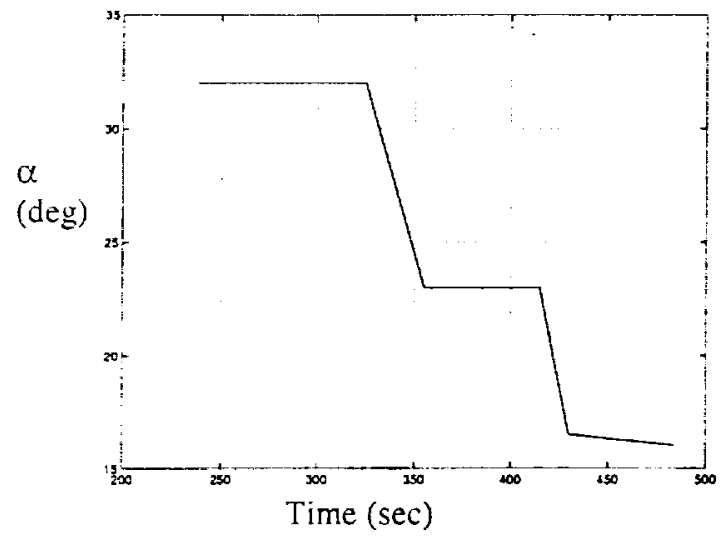

Figure 8 - Angle-of-attack vs. time

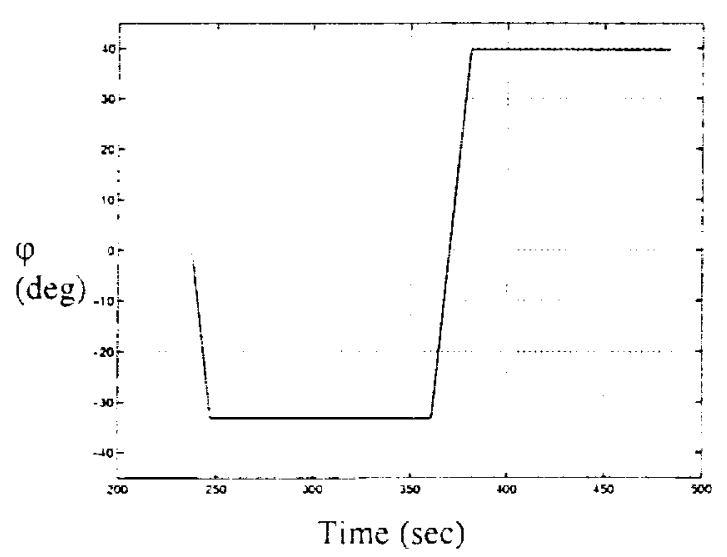

Figure 9 - Bank angle vs. time

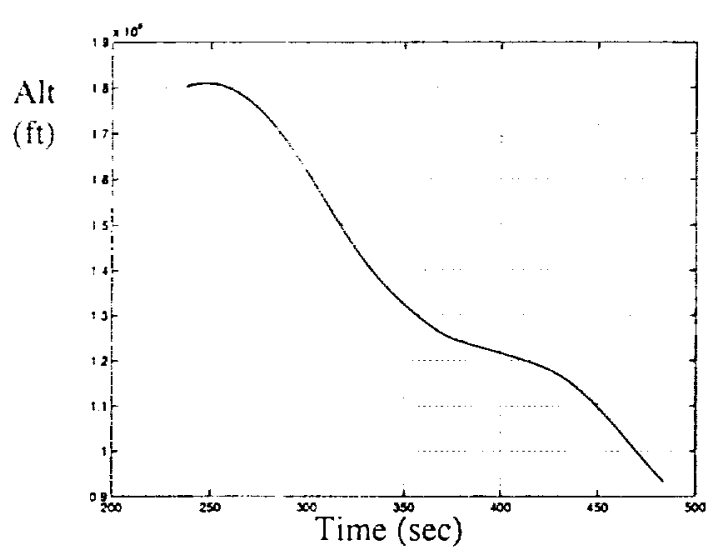

Figure $10-$ Altitude vs. time

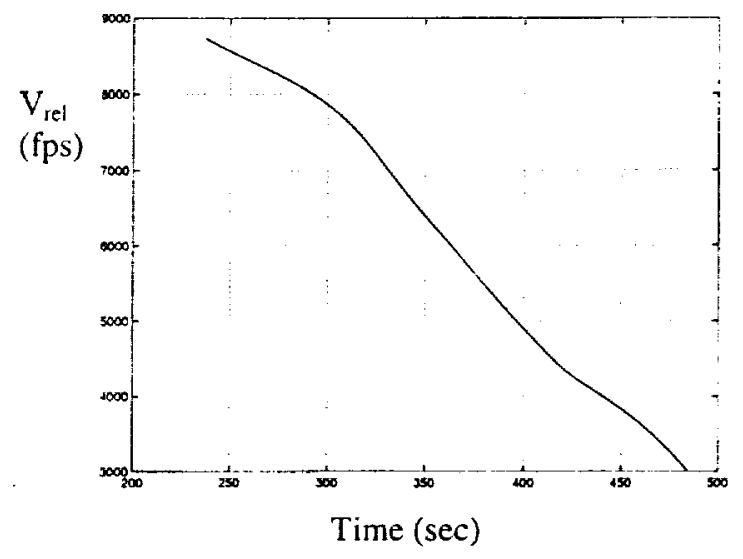

Figure 11 - Relative velocity vs. time

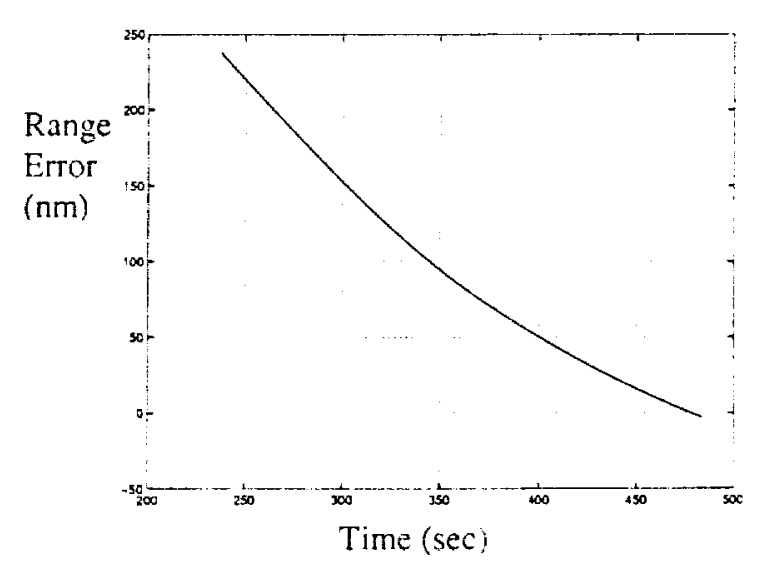

Figure 12 - Range error vs. time

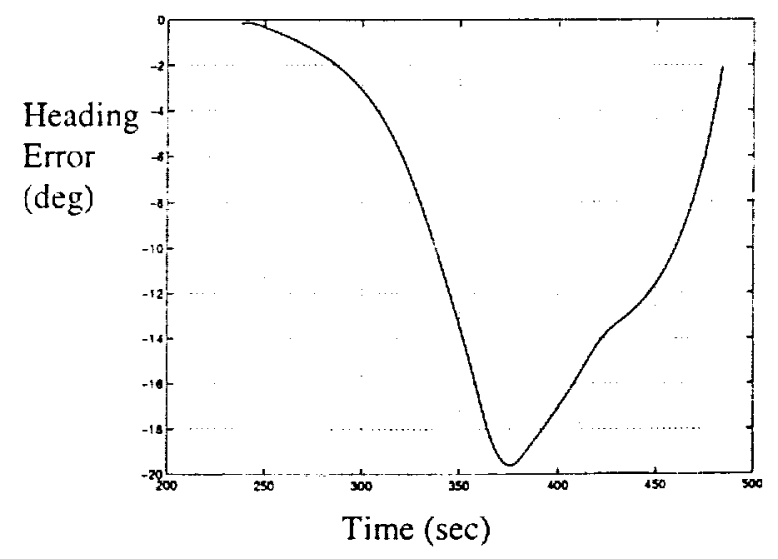

Figure 13 - Heading error vs. time 


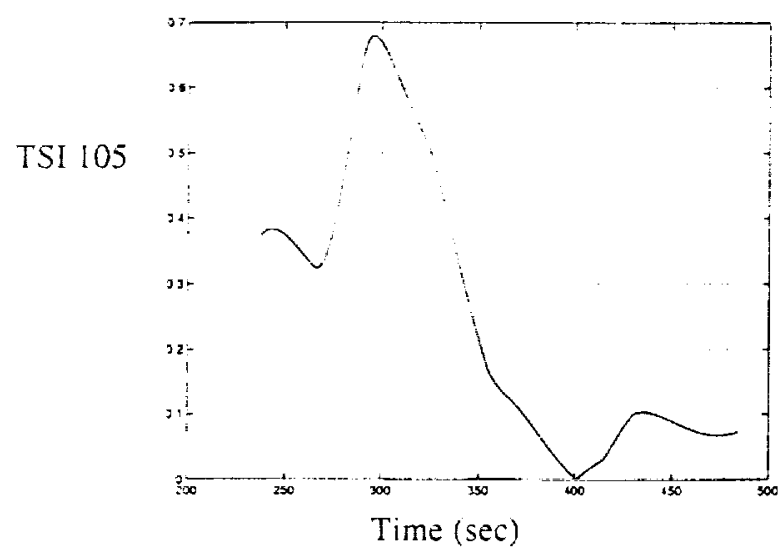

Figure 14 - TSI 105 vs. time
[3] Powell, R. W., "Numerical Roll Reversal Predictor-Corrector Aerocapture and Precision Landing Gudiance Algoithms for the Mars Surveyor Program 2001 Missions." AIAA-98-4574. August, 1998.

[4] Powell, R. W., "Six-Degree-of-Freedom Guidance and Control Entry Analysis of the HL-20." Journal of Spacecraft and Rockets, Vol. 30, No. 5, pp. 537-542, September-October, 1993.

[5] Harpold, J. C., and Graves, C. A. Jr., "Shuttle Entry Guidnace," Journal of the Astronautical Sciences, Vol. XXVII, No. 3, pp. 239-267, JulySeptember, 1979.

\section{CONCLUSIONS}

The results obtained from MATLAB simulation demonstrated successfully the applicability of using the predictor-corrector entry algorithm for the $\mathrm{X}$ 33/RLV operations. The next step is to demonstrate how efficiently this algorithm can be coded and used in 6-DOF MAVERIC test bed including Monte Carlo simulation. Another key factor in adopting the algorithm to real time application is establishing the initial solution guess as function of the perturbed initial conditions.

\section{ICKNOWLEDGEMENT}

The authors wish to acknowledge Dr. Patrick Rodi of Lockheed Martin Aeronautics Company - Palmdale for developing the TSI constraints subroutine and Greg Dukeman of NASA MSFC for his valuable suggestions and discussions during the development of the algorithm.

\section{REFERENCES}

[1] Hanson, J. M., "Advanced Guidance and Control Project for Reusable Launch Vehicles," AIAA-20003957. August 14, 2000.

[2] Hanson, J. M., et al, "Ascent, Transition, Entry, and Abort Guidance Algorithm Design for the X-33 Vehicle." AIAA-97-4409. August, 1997. 